
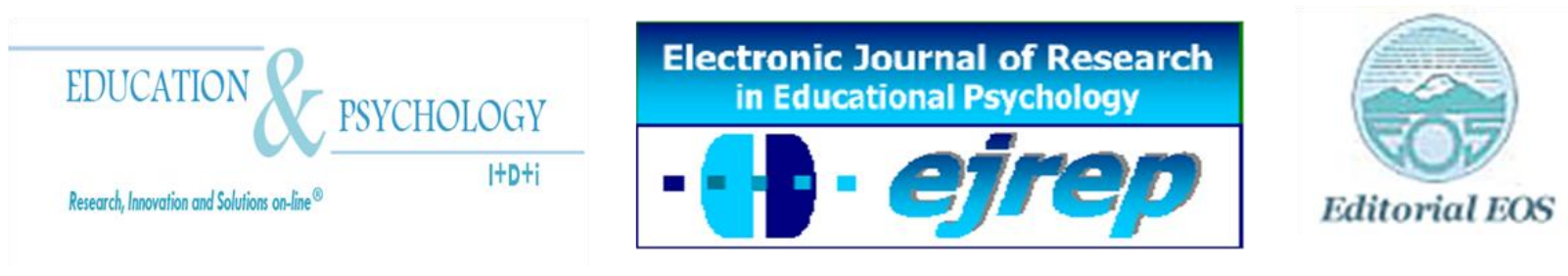

\title{
Metodología y Recursos del Maestro Itinerante de Audición y Lenguaje
}

\author{
José J. Carrión-Martínez y Antonio Luque de la Rosa
}

Departamento de Educación, Universidad de Almería

España

Correspondencia: José Juan Carrión Martínez. Universidad de Almería, Carretera de Sacramento s/n, 04120, La Cañada de San Urbano (ALMERÍA) E-mail: jcarrion@ual.es

(C) Education \& Psychology I+D+i and Editorial EOS (Spain) 


\section{Resumen}

Introducción. Habiendo transcurrido veinte años de andadura y desarrollo profesional desde el surgimiento del maestro de audición y lenguaje itinerante, parece conveniente reflexionar sobre el papel que ha venido desempeñando esta figura con objeto de aprehender las consideraciones oportunas que mejoren los planteamientos a adoptar de cara a promover la calidad de su actuación educativa. Con este estudio se pretende contribuir a la comprensión de la acción educativa de estos profesionales realizando un recorrido por los planteamientos metodológicos que la fundamentan, al tiempo que se analizan las estrategias organizativas y didácticas que llevan a cabo así como la tipología de recursos y materiales que utilizan.

Método. El objeto-problema de este estudio consistirá en realizar un análisis de la situación que viven los profesionales itinerantes de audición y lenguaje, profundizando en el estudio de la metodología y recursos que caracterizan la acción que vienen desarrollando. La investigación se ha llevado a cabo con una aproximación cualitativa a la acción de nueve maestros itinerantes de audición y lenguaje, repartidos en diferentes áreas geográficas. Dado el enfoque, el instrumento de toma de información prioritario ha sido la entrevista en profundidad, llevadas a cabo con una inmersión de campo de un año de duración. El análisis de los datos se ha desarrollado con estrategias de reducción, depuración, precategorización y categorización.

Resultados. Los resultados ponen de manifiesto que la metodología que desarrollan los maestros de audición y lenguaje itinerantes presenta una gran penetración de los modelos de intervención clínico-terapéuticos, con fuerte presencia de actividades y recursos focalizados en los componentes fonoarticulatorios.

Discusión y conclusiones. El eje principal de su actuación se centra en procedimientos reproductivos-repetitivos en los que predomina la actuación del docente; no se trabajan los contenidos del resto de las áreas ni se suelen encargar actuaciones para el aula ordinaria.

Palabras clave: Maestro, lenguaje, organización escolar, recursos, discapacidad. 


\title{
Methodology and Resources of the Itinerant Speech and Hearing Teacher
}

\begin{abstract}
Introduction. Having spent twenty years of business and professional development from the emergence of speech and hearing teacher traveling, it seems appropriate to reflect on the role he has been playing this figure in order to apprehend the things considered to improve the approach to adopt towards to promote the quality of its educational activities. This study aims to contribute to the understanding of the educational work of these professionals doing a tour of the methodological approaches that support it, while analyzing the organization and teaching strategies that perform and the types of resources and materials used.
\end{abstract}

Method. The object-problem of this study will conduct an analysis of the situation faced by mobile professionals for hearing and speech and deepen the study of the methodology and resources that characterize the action being carried out. The research now presented is within the ambit interpretive approach or paradigm, and that the nature of the offense under investigation, for the very purpose of the research activity, for how to address the analysis and interpretation of data and qualitative methodology from which we use for making information.

Results. The results show that the methodology developed by teachers of speech and hearing itinerant has a high penetration of clinical intervention models and therapeutic, with a strong presence of activities and resources focused on fonoarticulatorios components.

Discussion and conclusions. The main focus of its activities are focused on reproductiverepetitive procedures in which the predominant action of the teacher, not working the contents of other areas or activities are generally assigned to regular classrooms.

Keywords. Teacher, speech, school organization, resources, disability.

Received: 04/25/13 Initial aceptance: 06/23/13 Final aceptance: 07/15/13 


\section{Introducción}

La audición y el lenguaje son dos conceptos cuyos significados e interrelación sólo podrán entenderse en el contexto de un término más amplio como es la comunicación, cuyo análisis servirá para introducirnos en la confluencia de ambos. Como definió Dubois (1991), la comunicación es "la esencia misma de nuestra experiencia en el mundo y del mundo en nosotros: Comunicarse es ante todo creerse entendido y comprendido. Es imaginar que el otro nos responde" (p.46). Los seres humanos se comunican entre sí a través de diferentes medios y sistemas: los gestos, la música, las representaciones plásticas, los símbolos numéricos y gráficos, desempeñando la comunicación en nuestros días un papel mucho más importante que el tenido hasta ahora y, gracias al progreso técnico, adoptando formas y aspectos que no se habían podido ni siquiera soñar. Este desarrollo ha planteado nuevos problemas, no sólo para el estudio de la comunicación en sí misma, sino también, y de una manera particular, para el estudio de nuestro principal medio de comunicación: el lenguaje (Wigg, 2001).

La importancia del lenguaje radica en tener, además de una función comunicativa y expresiva, un papel importantísimo en la elaboración del pensamiento (Crystal, 1981; Monfort, 1983, 1990; Vigotsky, 1995). El desarrollo de la inteligencia, y por extensión de la personalidad del hombre, está supeditado al lenguaje que actúa como cauce del complejo entramado de abstracciones que se suscitan en nuestro cerebro (Cooper, Moodley y Reynell, 1982). Si bien han venido existiendo en nuestro país perfiles profesionales dedicados a la atención específica de problemáticas relacionadas con la pronunciación y el habla (logopedas), surge en España al inicio de la década de los noventa una nueva figura docente: el maestro de audición y lenguaje (en adelante, AL).

En su propia denominación se aprecia el deseo de cambio en dicho rol, promoviendo la superación de los planteamientos recuperadores y rehabilitadores de carácter terapéutico que afectan a determinados canales de comunicación (audición/fonación), para avanzar hacia una concepción de dicho profesional como potenciador del desarrollo comunicativo, cognitivo y sociopersonal a través del lenguaje en el conjunto del alumnado.

\section{Modelos existentes: la perspectiva multidimensional}

Se pueden realizar diferentes sistemas de clasificación de las opciones de intervención según los parámetros y criterios que se consideren. Así, Gallardo-Ruiz y Gallego-Ortega 
(1993) señalan inicialmente cuatro modelos de intervención en la faceta lingüísticacomunicacional:

a) Modelo lingüístico. Considera que existe una incapacidad lingüística independiente que debe ser evaluada y tratada desde una perspectiva linguística. Ante este planteamiento se distingue un enfoque formal (preocupado por establecer las estructuras sintácticas, relaciones semánticas y oposiciones fonológicas) y otro más funcional (perspectiva más pragmática en ambientes naturales).

b) Modelo conductual. Entiende el programa como una conducta a la que aplica los mismos principios de la psicología que a otros comportamientos.

c) Modelo cognitivo. Defiende la existencia de una íntima relación entre pensamiento y lenguaje.

d) Modelo clínico. Utiliza terapias psicoanalíticas para intervenir sobre los síntomas y la etiología de las dificultades.

Frente a la parcialidad de modelos más o menos biomédicos (causas) o conductuales (síntomas) (Bloom y Lahey, 1978) que centran la atención en una faceta del problema, la actual visión multidimensional y ecológica del hecho educativo y del ser humano y el perfil del maestro de audición y lenguaje nos hace inclinarnos por un modelo ecléctico como el más idóneo para abordar el desarrollo del lenguaje en el ámbito escolar, inspirándose en las teorías sociointeractivas, que aceptan la diversidad como punto de partida, y cuyo objetivo principal es el desarrollo global del lenguaje (Lindsay y Dockrell, 2002; Castejón-Fernández y EspañaGanzaráin, 2004; Acosta-Rodríguez, 2005, 2006a). Dicho modelo parte de la concepción del apoyo curricular, que se preocupa no sólo de atender al alumno que presenta la dificultad, sino de otros puntos dentro de la comunidad educativa (Prelock, 2000; Lindsay y Dockrell, 2002; Castejón-Fernández y España-Ganzaráin, 2004; Acosta-Rodríguez, 2005, 2006b).

Este modelo integrador responde a un criterio de funcionalidad del tratamiento, adquiriendo una vertiente holística y global que selecciona aquellos planteamientos más convenientes para orientar un específico proceso de intervención. Se parte de una valoración integral de las aptitudes del alumno en todos sus ámbitos de desarrollo, con el objeto de comprobar sus potencialidades y alteraciones tanto a nivel cualitativo como cuantitativo, concibiéndolas más como dificultades situadas en un contexto que como déficits vinculados al individuo (Acosta-Rodríguez y Moreno-Santana, 2003). 
En cuanto a la temporización de la intervención, como criterio general se aconseja la actuación tan pronto como se aprecie la dificultad o se perciba inicialmente la posibilidad de su manifestación, estableciendo procedimientos sistemáticos de aprendizaje en el ámbito comunicativo, lingüístico y prelingüístico (Acosta-Rodríguez y Moreno-Santana, 2003). Siendo conocedores de la correlación existente entre éste y el desarrollo cognitivo o afectivosocial, se vuelve relevante aunar esfuerzos no sólo asistenciales sino de carácter preventivo. Desde esta perspectiva, un tratamiento educativo no es simplemente una intervención logopédica para que el alumno consiga una conducta lingüística en la sesión, sino para que pueda utilizar y servirse del lenguaje en los distintos contextos y circunstancias de su vida escolar, familiar y social, debiendo considerar en su plan de actuación el conjunto de variables que conforman dicho entorno (Acosta-Rodríguez, 2006b; Hemmeter y Grisham-Brown, 1997).

Al confirmar como idónea esta perspectiva multidimensional, es esencial que los tratamientos en audición y lenguaje se coordinen lo más estrechamente posible con el trabajo general del aula, al ser éste el contexto donde el alumno pasa la mayor parte del tiempo y donde se encuentran mensajes e interlocutores más significativos para su mundo. Es fundamental promover la participación activa del alumno, su naturalidad y espontaneidad en el proceso de aprendizaje, potenciando así su carácter funcional (Acosta-Rodríguez, 2003, 2004, 2005; Richards y Rodgers, 1986).

Estos planteamientos exigirían del maestro de audición y lenguaje sólidos fundamentos sobre el desarrollo del lenguaje y la comunicación, suficiente formación psicopedagógica, experiencia y trabajo personal, toma de conciencia de las propias posibilidades y limitaciones, rigurosos controles sobre la práctica, talante integrador y asertividad (Gallardo-Ruiz y Gallego-Ortega, 1993). Además de encontrar clasificaciones de modelos de intervención como la anterior, en función de la fundamentación del desarrollo lingüístico, encontramos otras que se centran en los diversos modelos organizativos de apoyo aplicable a la faceta lingüística-comunicativa. Como síntesis de las principales características de dichos modelos presentamos las siguientes (Ver tabla 1): 
Tabla 1. Modelos organizativos de apoyo educativo (Adaptado de Parrilla-Latas, 1996, p.83)

\begin{tabular}{|c|c|}
\hline $\begin{array}{c}\text { MODELOS DE } \\
\text { APOYO }\end{array}$ & CARACTERÍSTICAS \\
\hline $\begin{array}{c}\text { Apoyo } \\
\text { Terapéutico }\end{array}$ & $\begin{array}{l}\text { - "Un apoyo centrado en el niño, guiado y dirigido por especialistas o expertos, } \\
\text { basado en las dificultades atribuidas a ese niño” (Parrilla-Latas, 1996, p.84) } \\
\text { - El papel del tutor no es activo. El experto se encarga del apoyo. } \\
\text { - Se trabaja en el aula de apoyo (¿descanso para tutores?). } \\
\text { - Apoyo individual que se centra en las dificultades de cada niño e interviene } \\
\text { impartiendo las enseñanzas apropiadas a sus necesidades (Muntaner-Guasp, 2000). }\end{array}$ \\
\hline $\begin{array}{c}\text { Apoyo } \\
\text { Colaborativo } \\
\text { Individual }\end{array}$ & $\begin{array}{l}\text { - La atención deja de ser "experta" para tener en la colaboración el referente de la } \\
\text { actuación. } \\
\text { - Para que sea real, es necesario redefinir los roles de los profesores promoviendo } \\
\text { una comunicación e implicación real de los mismos. } \\
\text { - No obstante, sigue incidiendo en las dificultades de los alumnos sin cuestionar la } \\
\text { participación de la escuela o los factores contextuales. }\end{array}$ \\
\hline $\begin{array}{l}\text { Apoyo de } \\
\text { Consulta/ } \\
\text { Recursos }\end{array}$ & $\begin{array}{l}\text { - Se asume la relación de las necesidades de los alumnos con elementos del } \\
\text { contexto. } \\
\text { - El ámbito de actuación de apoyo trasciende a factores escolares y sociales. } \\
\text { - Sin embargo, se prima la intervención técnica o de algún experto a quien } \\
\text { consultar las respuestas que precisa el centro en su conjunto. }\end{array}$ \\
\hline $\begin{array}{l}\text { Apoyo } \\
\text { Curricular }\end{array}$ & $\begin{array}{l}\text { - Se redefinen los perfiles profesionales (incluidos los del maestro de apoyo) al } \\
\text { trabajar en una "colaboración para resolver problemas institucionales, grupales de } \\
\text { aula o individuales" (Parrilla-Latas, 1996, p.106). } \\
\text { - Los destinatarios del apoyo no son sólo los niños sino el conjunto de la } \\
\text { comunidad, siempre desde un plano colaborativo. } \\
\text { - Posibilita una real integración de los alumnos con necesidades educativas } \\
\text { especiales en el aula ordinaria al fundamentarse en la cooperación del conjunto de } \\
\text { profesionales desde la adaptación de un currículo común. } \\
\text { - Concepción preventiva y de desarrollo del apoyo (no sólo correctiva) y asunción } \\
\text { de la diversidad como un valor enriquecedor para todos. } \\
\text { - Implica una cultura educativa "peculiar" ya que las barreras de la integración } \\
\text { están en las personas, en su cabeza y en su corazón. }\end{array}$ \\
\hline
\end{tabular}

De las opciones de intervención comentadas, en el perfil profesional del maestro de audición y lenguaje advertimos la importancia de apostar hacia actuaciones colaborativas integrales próximas al modelo de apoyo curricular (equipo de profesionales y familia) y ecológicas (actos de comunicación espontánea, funcional y generalizable en los distintos contextos y circunstancias de su vida escolar, familiar y social) que refrendan la importancia del contexto escolar, todo ello desde un planteamiento integrador de la actuación educativa. Así, la actuación sobre el alumnado para el adecuado desarrollo lingüístico no es privativa del maestro de audición y lenguaje, debiendo ser compartida por todos los profesores del centro en aras a favorecer la optimización de las ocasiones que se ofertan en la clase como contexto, favoreciendo la comunicación y la interrelación entre dichos alumnos y los demás compañeros y aumentando así su confianza y autoestima (Moya-Maya, 2002). 
$\mathrm{Si}$ realizamos un recorrido por las diversas investigaciones nacionales e internacionales realizadas en torno a esta temática, encontramos aportaciones como las que señalan que el adecuado desarrollo lingüístico en el aula sólo se conseguirá mediante una metodología que promueva en el alumnado la actuación combinada sobre los diversos componentes lingüísticosa a través de estrategias colaborativas en las que el papel del maestro de audición y lenguaje es clave a la hora de coordinar y asesorar el papel de los demás educadores (Acosta- Rodríguez, 2004, 2005; Acosta-Rodríguez y Moreno Santana, 1999, 2003).

Con relación al primer aspecto, existen estudios que remarcan la necesidad de incidir en el conjunto de factores promotores de un adecuado desarrollo lingüístico -ámbito psicomotor, emocional, socioafectivo y cognitivo- (Fey, 1986; Fey, Catts y Larrive, 1995), aportando propuestas más detalladas en cuanto a la forma de trabajar en el aula en las que aparece explícitamente la necesidad de desarrollar como contenidos ciertos prerrequisitos del lenguaje y desarrollar la faceta cognitiva y socializadora de dicha promoción lingüística. Así, cabe señalar las aportaciones de McCauley y Fey (2006) quiénes después de más de 20 años de investigación básica y aplicada nos proponen un modelo de intervención estructural que incorpora ciertos aspectos novedosos relacionados con la frecuencia y la intensidad de las sesiones de trabajo; simultáneamente, se insiste en la importancia de la reevaluación de la intervención y la consecuente modificación de los programas.

En estos modelos, se partiría de un enfoque de intervención integral dentro del aula que se ocupara de conseguir objetivos comunicativos y lingüísticos, no tanto a partir de un entrenamiento en habilidades aisladas, sino dentro de actividades significativas para el alumnado, es decir, dentro de las rutinas y actividades desplegadas en el aula a las que el alumnado debe enfrentarse haciendo uso del lenguaje (Acosta Rodríguez, 2004, 2005; Acosta Rodríguez y Moreno Santana, 1999, 2003). En esta línea, frente a planteamientos altamente estructurados que recurren a procedimientos como la imitación, el moldeamiento, el desvanecimiento y la generalización, pretendiendo conseguir predominantemente objetivos relacionados con habilidades articulatorias (Hodson y Paden, 1991) o morfosintácticas (Fey y Cleave, 1997), un enfoque más global e interactivo del lenguaje se centraría en facilitar la comunicación social por medio del modelado interactivo, recurriendo a veces a conversaciones o narraciones para conseguir objetivos lingüísticos (Brinton, Fujiki y Powell, 
1997) y todo ello ahondando en la perspectiva colaborativa con el conjunto de profesorado y la familia.

Otras investigaciones refrendan dicha perspectiva, apostando por un enfoque curricular cercano a lo que sería una atención generalizada del lenguaje y la comunicación en el conjunto del alumnado, caracterizándose dicha actuación por su sentido dinámico, multidimensional e interactivo en el conjunto de los componentes del lenguaje (Moreno Santana y Leal Hernández, 2005), debiendo abarcar no sólo la faceta correctiva o rehabilitadora sino su carácter preventivo y de desarrollo.

En cuanto al papel colaborativo de padres y resto de profesores, existen estudios que evidencian los resultados positivos que supone la realización de actividades en el aula ordinaria y en casa complementarias a las ejecutadas en el aula de apoyo por parte del profesional especializado, trasladándose ahora el foco de la intervención directamente al niño. En un metaanálisis llevado a cabo por Law, Boyle, Harris, Harkness y Nye (2000) se señalan hasta un total de 33 trabajos en los que se pone en evidencia la bondad de esta modalidad de intervención sobre el lenguaje expresivo de los niños y niñas, potenciando las prácticas centradas en la familia y el profesorado con carácter colaborativo, en los que se parte de una intervención temprana apoyando y fortaleciendo todo el contexto familiar y escolar del niño y promoviendo la participación activa de dichos agentes en la toma de decisiones, mediante la realización de propuestas individualizadas y diferenciadas. Entre los programas más utilizados, dentro de esta corriente, destaca considerablemente el Programa Hanen, creado por Ayala Manolson (Manolson, 1992), y vinculado a una perspectiva socio-interaccionista de adquisición del lenguaje. El objetivo principal es la enseñanza explícita a los padres y profesores de una serie de procedimientos destinados a mejorarlas situaciones de interacción con sus hijos y alumnos que potencien la interacción comunicativa.

\section{Objetivos}

Deseamos indagar sobre las metodologías y recursos de intervención del maestro de audición y lenguaje itinerante, los factores que la condicionan, características y modelos que proyectan. En concreto:

1. Conocer si la tipología de actividades que llevan a cabo conlleva la realización de actuaciones de carácter preventivo y de desarrollo además de las históricamente terapéuticas realizadas por los logopedas. 
2. Analizar si la actuación atiende la totalidad de componentes lingüísticos (fonético, morfosintáctico, semántico y pragmático) desde una dinámica colaborativa, plenamente incardinada en el currículo, que potencie el aspecto socializador y educativo en el conjunto del alumnado.

\section{Método}

\section{Participantes}

En la investigación que presentamos, la población hace referencia al conjunto de maestros itinerantes de audición y lenguaje, figura profesional que puede considerarse de un perfil muy exclusivo de España, con relación a la cual intentaremos analizar y comprender su actuación profesional, acercándonos a la práctica educativa que realizan, viviéndola y percibiéndola en su singularidad, con objeto de situar significativamente el conjunto de dimensiones que conforman esa realidad natural. Se ha tomado una selección de informantes formada a partir de un conjunto de 9 maestros especialistas en audición y lenguaje, que constituyen en el momento de la investigación la totalidad de los AL adscritos a la plantilla de Equipos de Orientación Educativa (en adelante, EOE) de Almería (España). Ver tablas 2 y 3.

Tabla 2. Número de sesiones de contacto con cada maestro de audición y lenguaje

\begin{tabular}{clllllllll}
\hline $\mathrm{AL}$ & 1 & 2 & 3 & 4 & 5 & 6 & 7 & 8 & 9 \\
\hline $\mathrm{N}^{\mathrm{o}}$ contactos & 6 & 5 & 5 & 5 & 5 & 5 & 6 & 4 & 5 \\
\hline
\end{tabular}

Tabla 3. Siglas identificativas de cada tipo de informante

\begin{tabular}{ccccccc}
\hline AL & CC & DD & Ma & Or & PT & TU \\
\hline $\begin{array}{c}\text { Maestro de } \\
\text { audición y } \\
\text { lenguaje }\end{array}$ & $\begin{array}{c}\text { Coordinador } \\
\text { de Equipo de } \\
\text { orientación } \\
\text { educativa }\end{array}$ & $\begin{array}{c}\text { Miembro de } \\
\text { equipo }\end{array}$ & Madre & Orientador & Maestro de & Maestro \\
& & & & pedagogía & tutor \\
\end{tabular}

\section{Instrumentos}

Se han utilizado instrumentos propios de la metodología cualitativa: entrevista en profundidad (en los resultados se codificará con E), observación de campo (en los resultados se codificará con CC) y análisis de documentos (en los resultados se codificará con $\mathrm{AD}$ ):

1) La entrevista en profundidad se ha configurado con un planteamiento abierto, sin un cuestionario-guía como tal, ya que las entrevistas se han contextualizado en una inmersión en los centros, tomandose como referencia de guía por parte de los investigadores los 
objetivos de la investigación, siempre con una prioridad a la libre construcción del discurso por parte del entrevistado y mínimas reconducciones por parte del entrevistador para favorecer la aparición de información relevante.

2) Más abierta aún ha sido la observación de campo, pues se ha llevado a cabo primando la inmersión natural en las aulas de audición y lenguaje implicadas en el desarrollo de la investigación, con declarada, expresa y compartida con los maestros la facción de obervación, trasladando después de forma minuciosa al cuaderno de campo la información contingente con relación a los objetivos.

3) El análisis de los documentos de los maestros de audición y lenguaje y de los equipos de orientación educativa participantes, tanto de planificación como de evalaución y memoria, ha sido realizado para la obtención de información añadida, así como para la triangulación metodológica de la información aportada en entrevistas y observación.

\section{Procedimiento}

El procedimiento se ha sustentado en una inmersión múltiple en el campo, con nueve focos, a los que se llega con negociaciones individuales para cada foco. Durante la inmersión se han alternado las entrevistas en profundidad con diálogos espontáneos con distintos actores que se han ido registrando en el cuaderno de campo, a la vez que se acopiaban documentos para su posterior análisis.

Los nueve focos, consitituídos por maestros itinerantes de audición y lenguaje, ubicados en siete equipos de orientación educativa de la provincia de Almería (España), han sido abordados como contextos de inmersión durante periodos de seis semanas con un día, sesión escolar completa, de estancia semanal con cada de ellos. Se descartaron los meses de septiembre y octubre para que la inmersión coincidiese con un periodo de actuación de los AL ya exento de las incertidumbres organizativas de inicio de curso frecuentemente presentes en la atención itinerante. Este planteamiento generó cuatro contextos de inmersión: dos contextos constituidos por los dos EOE que tenían dos AL en su plantilla, y otros dos contextos de dos y tres AL cada uno, agrupándolos por criterios de proximidad geográfica para facilitar la operatoria del propio proceso de inmersión. Eso llevó a un período de inmersión total de 24 semanas efectivas, lo que permitió terminar antes de junio, y así eludir un tiempo con un nivel de incidencias organizativas y curriculares generalmente más alto. 
En términos de sesiones eso ha conducido a tener con varios de ellos las seis sesiones de contacto, otro grupo con alguna incidencia cinco y en un caso cuatro. En todos los casos la aproximación al AL, se articuló de forma personal y directa, así como simultánea a la gestión ante el equipo directivo, dentro del marco de colaboración que el grupo de investigación de pertenencia mantiene para diversas actividades con la Delegación de Educación de la provincia de Almería. Tras el período de inmersión se procedió al tratamiento de la información, con alguna visita adicional posterior para confrontar datos.

\section{Diseño y análisis de datos}

El análisis e interpretación de los datos cualitativos de los que nos servimos para la toma de información (Goetz y LeCompte, 1988; Rodríguez-Gómez, Gil-Flores y GarcíaJiménez, 1996; Stake, 2007; Taylor y Bogdan, 1986; Woods, 1989), en una aproximación etnográfica de caso múltiple se ha desarrollado en varias etapas:

a) Acopio y transcripción de la información.

b) Reducción y depuración de información desde el criterio marcado por los interrogantes y objetivos.

c) Precategorización y construcción texto interpretativo.

d) Simplificación textual y de precategorías.

e) Categorización e informe final.

En el tratamiento de la información no se ha considerado pertinente el tratamiento a través de programas informáticos, pues se trataba de información siempre tomada en contexto de inmersión, tanto la de la entrevista como la de la observación, que requería un análisis interpretativo con un fuerte peso de la capacidad del propio investigador para su mejor aprovechamiento. De hecho, el mapa de categorías generado, no parte de un análisis precategorial, sino de la interpretación constructiva de la información y del conjunto de elementos didácticos y linguísticos que el propio estado de la cuestión nos alumbraba (ver Tabla 4):

Tabla 4. Categorías

\begin{tabular}{cc}
\hline & CATEGORÍAS \\
\hline Didácticas & lingüísticas \\
\hline Metodología & Componente fonético-fonológico \\
\hline Actividades & Componente semántico \\
\hline Implicación tutorial & Coponente morfosintáctico \\
\hline Organización temporal & Componente pragmático \\
\hline Organización espacial & \\
\hline Recursos materiales & \\
\hline
\end{tabular}




\section{Resultados}

Rasgos generales de su metodología didáctica

Las características más relevantes son:

1) Según hemos apreciado, no aparece en los documentos un modelo definido, sino indicaciones de los tipos de actividades y recursos que vienen desarrollando estos profesionales (AD, AL7). ${ }^{1}$

2) Ensayan procedimientos y actividades que acercan su labor inicialmente terapéutica a las intenciones educativas que todo diplomado en magisterio ha vivenciado a lo largo de su formación inicial (CC, AL5).

- "Se nota que se desvive en intentar rentabilizar al máximo su labor dotándola del mayor tinte educativo posible” (E, DD).

3) Sus actuaciones suelen ser motivadoras para el alumnado y pretenden fomentar su participación. Según describen estos profesionales, intentan que resulten amenas y procuran utilizar un material que sea -“muy variado y atractivo para los chavales. Ellos [el alumnado] vienen contentos y les gusta lo que alli hacen: juegos, fichas, etc.”- (E, Tu).

- "Mi metodología, bueno, pues yo intento que los niños se lo pasen bien cuando vienen conmigo. Yo en primer lugar los valoro, me planteo unos objetivos y a partir de ahí intento conseguirlos de la forma más amena posible para ellos y para mi." (E, AL7)

4) Suele realizar las actividades con los alumnos uno a uno o en parejas no encontrando suficiente apoyo y coordinación con el tutor en las tareas del aula:

- “Es muy importante, pero haría falta más tiempo de todos para coordinarse y la verdad es que vamos siempre apresurados. Ya he comentado que las reuniones son esporádicas aprovechando los recreos. Además ella no dispone

\footnotetext{
${ }^{1}$ Las citas textuales de datos empíricos de carácter cualitativo, se realiza en la parte de resultados utilizando un par de claves entre paréntesis, por ejemplo: (E, AL7). El primer componente del par es una letra mayúscula que representa la instrumento que da origen al dato $(\mathrm{E}, \mathrm{CC}$ ó $\mathrm{AD})$, y el segundo componente del par representa al informante como los tipifica la Tabla 3, con la particularidad de que si se trata de un AL se le añade el número del 1 al 9 que los diferencia entre si (Tabla 2).
} 
de un horario específico de dedicación al centro para reuniones y es la primera que siempre lleva prisa” (E, Tu)

5) Cabe establecer cierta diferenciación con relación a los métodos didácticos utilizados por estos profesionales en función del componente lingüístico a desarrollar. La metodología de estos profesionales -según el componente lingüístico que se trabaje-, se mueve entre la recuperación conductual/formal del lenguaje y el desarrollo de una conciencia sistemática de las funciones del mismo, no existiendo un procedimiento propio y organizado de actuación generalizado, sino la utilización de materiales elaborados o del mercado en función de la problemática específica, periodicidad de actuación e idiosincrasia de cada profesional (CC, AL3); (CC, AL5).

\section{Tipología de actividades}

Con relación a la tipología de actividades que desarrolla, dado que tanto las problemáticas fonético-fonológicas como las del resto de componentes suelen demandar actuaciones lingüísticas diversas (praxias, discriminación, articulación, memoria, fluidez, identificación, etc.), para determinar las actividades precisas dichos profesionales suelen recurrir a los resultados de las pruebas de exploración (CC, AL1), siendo sus resultados orientadores tanto del tipo de actividades a desarrollar (materiales editados o propios) (AD, AL2), como del seguimiento y valoración de logros adquiridos al final del curso (hoja de seguimiento y valoración final).

\section{Componente fonético-fonológico}

En el caso de este componente fonético-fonológico, la secuencia de actividades suele caminar desde el desarrollo de los requisitos previos de emisión o moldeamiento (respiratorios, soplo, logocinéticos), pasando por ejercicios de posicionamiento y emisión aislada e integrada (lenguaje repetido modelado mediante la progresiva atenuación de ayudas como depresores, palillos, silbatos, espejo, matasuegras, etc.), para concluir con la promoción del lenguaje dirigido (lotos) y espontáneo (diálogos), (AD, AL8); (CC, AL9).

La mayoría de las actividades parten de situaciones lúdicas (juegos) intentando mantener la atención del alumnado ante tareas a veces tediosas pero, en opinión de estos profesionales, - "imprescindibles, como listados de palabras con los que hay que 
machacarles"-, (E, AL4), ejercicios de respiración/soplo, relajación o praxias (muy frecuentes en las dislalias), etc. (CC, AL7).

- "Que no sean monótonos ni repetitivos, ya que la logopedia es pesada de recuperar y los ejercicios son cansinos para los críos.” (E, DD).

En términos generales, los restantes componentes se caracterizan por actividades muy variadas en función de la información aportada por los informes logopédicos, intentando resolver las problemáticas apuntadas en los mismos mediante ejercitaciones o actuaciones específicas que aparecen programadas en un bloque conjunto en los documentos de planificación de estos profesionales (AD, AL3).

\section{Componente semántico}

Las actividades para trabajar el componente semántico (referente al significado) se fundan en juegos y software de identificación/fragmentación de palabras así como de enriquecimiento de vocabulario (AD, AL2).

\section{Componente morfosintáctico}

Para trabajar el componente morfosintáctico (formación de las frases) el maestro AL utiliza diversas actividades con las que pretende reforzar a los alumnos en los procesos de lectura y escritura, tratando de corregir los errores que cometen en estas habilidades instrumentales: sustituciones de unos grafemas por otros, inversiones en el orden de las sílabas, rotaciones de las letras, omisiones, adiciones, fragmentaciones de palabras, uniones incorrectas...calidad del grafismo (tamaño, letra, adaptación a la pauta...) y en la lectura bloqueos, repeticiones y retrocesos, sustituciones fonológicas, falta de ritmo, falta de comprensión, etc. (AD, AL3). Estas actividades, dada su amplitud de facetas y cercanía a lo trabajado en el aula ordinaria, "se suelen llevar a cabo sólo en casos de complemento autónomo de sesión para los alumnos más aventajados”(E, AL5), al ser consideradas como propias del área de lengua o del maestro de apoyo y no específicas del AL (CC, AL5).

\section{Componente pragmático}

El componente pragmático (en el caso de que el grupo lo necesite) se trabaja mediante actividades en las que participa conjuntamente el grupo. Estos periodos de estimulación de la comunicación oral persiguen el enriquecimiento de la misma en algunos alumnos que por 
diversas causas (inmadurez, falta de estimulación u otras) presentan un lenguaje muy inmaduro y desfasado con su edad (CC, AL3).

Para las actividades desarrolladas hacia este componente, se utiliza con frecuencia el diálogo en el grupo y la expresión de experiencias propias, aprovechando la intimidad del pequeño grupo para que el niño más inhibido pueda comunicarse y de esta forma crecer también socialmente (CC, AL3). Otras veces, cuando los casos lo requieren, suele narrar cuentos e incluso representarlos con guiñol, disfraces o gestos, para trabajar la secuencia temporal de hechos e invitar a narrar, describir, representar... (CC, AL3).

\section{Implicación de los tutores}

En cuanto a la implicación de los tutores en el desarrollo de las actuaciones que los maestros de audición y lenguaje desarrollan con los alumnos suele ser escasa, promoviendo que los profesionales objeto del presente estudio mantengan una labor de carácter individualista al ser programada y ejercida -en la mayor parte de los casos- con total independencia del resto del profesorado que atiende a dicho alumno:

- “No conozco sus planificaciones" (E, DD)

- "Considero que lo interesante es potenciar la actuación directa de estos profesionales ya que los maestros del aula ordinaria no tenemos tiempo ni preparación para atajar esos problemas (y tenemos otros niños que reclaman al mismo tiempo nuestra atención)” (E, Tu).

Si alguna vez entran en las aulas o charlan con el profesorado, intentan servir de modelos de actuación y les ofrecen algunas orientaciones generales pero reconocen que se necesitaría una actuación conjunta y continuada AL/Tutores/Familia (CC, AL1). "Se necesitaría un sistema de coordinación entre todos que no existe. Realmente, si no están en el centro no podemos pedirles más. Van siempre corriendo...” (E, DD). Aunque no es frecuente, a veces aparecen casos de profesores que manifiestan interés en facilitar el enriquecimiento lingüístico de los alumnos diagnosticados y del conjunto de la clase, si bien al avanzar el curso suelen irse apagando dichas iniciativas (CC, Tu).

Los profesores de aula de apoyo a la integración pueden ser la otra cara de moneda en este aspecto, al mostrar una colaboración más continuada y coincidir con los intereses propios de la adecuada atención a la diversidad en el ámbito educativo (CC, AL9); (CC, PT): 
"Con los únicos maestros que suelen mantener alguna colaboración es con los profesionales de Pedagogía Terapéutica, pero con el profesorado de aula, en pocos casos." (E, PT)

\section{Organización temporal}

Otro aspecto a comentar es la organización temporal en que desarrollan sus actividades. Así, algunos profesionales comentan la secuencia de actuaciones diarias improvisadas como la estrategia más idónea tras los largos periodos carenciales que tienen en algunos casos establecidos de manera sistemática entre sesión y sesión, o bien por causas sobrevenidas de absentismo del alumnado (CC, AL8).

Con relación a la distribución horaria asignada inicialmente, en opinión de estos profesionales, además de ser insuficiente el tiempo de atención global a los centros para desarrollar una función educativa integradora, colaborativa y global, se necesita -“un mínimo -como hemos dicho muchas veces en las reuniones-, de dos sesiones semanales de treinta minutos en las que tú puedas hacer un seguimiento a ese niño"- (E, AL8) de cara a conseguir los objetivos logopédicos que se vienen trabajando, periodicidad que no siempre se alcanza.

- "La logopedia necesitaría una mayor atención, no una vez a la quincena, -eso si no falta por motivos justificados-, ya que así no se consigue gran cosa. Además se está atendiendo una parte mínima de los casos requeridos.” (E, DD).

Con la escasa periodicidad asignada en la actualidad es imposible llevar a cabo un seguimiento ni tan siquiera de corte individualista logopédico, demandando por parte del colectivo la necesidad de implantar como norma un mínimo de atención de dos sesiones semanales para impulsar la mejora en el tratamiento.

- “Ahora mismo, el tiempo de atención que damos en función de los medios de que disponemos es muy poco. Una atención logopédica requiere de las dos a tres sesiones semanales. Lo que pasa es que ahora mismo, en una zona amplia, con muchos centros y no se puede dar más atención.” (E, AL5)

-“Dedicar más horas al centro para así poder realizar su función con eficacia. Con la cantidad de niños diagnosticados que hay y el poco tiempo semanal que viene, la labor de logopedia deja mucho que desear." (E, Tu) 


\section{Organización espacial}

1) Con relación a la organización espacial en que desarrollan su actuación, la práctica totalidad de los AL considera que su intervención se realiza predominantemente fuera del aula ordinaria (C, AL4), con alumnado de forma individual o en pequeño grupo, de características lingüísticas similares (los alumnos de necesidades educativas especiales de aula específica siempre asisten solos al requerir -según comentan-, un seguimiento más continuo e individual):

- "Ella se lleva los niños a la biblioteca y alli les trabaja sus dificultades.” (E, $\mathrm{Tu})$.

- "Ella se lleva a mi hija a un pequeño aula que está junto a la clase de los apoyos. El trabajo lo realiza ella (...) va cogiendo a los alumnos uno a uno por orden." (E, Ma).

2) Sobre el tipo de agrupamiento, hay quien señala que a veces se opta por el pequeño grupo con objeto de dar respuesta a la gran demanda, pero al mismo tiempo afirman que en logopedia trabajar en grupo no sirve para nada (CC, AL7).

- "Para que sea efectivo el tratamiento debería ser de uno en uno o como mucho de dos en dos" (E, AL5).

Otros profesionales, en cambio, reconocen que el trabajo en el aula ordinaria resultaría muy positivo al potenciar la faceta comunicativa y la interacción social con el grupo natural (CC, AL1), declarando realizar algo en Infantil para evitar que algunos alumnos muy pequeños se despeguen de su clase (CC, AL5).

3) Si analizamos la utilización didáctica del espacio del aula, podemos apreciar las siguientes consideraciones en relación con una sesión-tipo:

- Una vez constituido el pequeño grupo de trabajo para la sesión del AL, el alumnado que lo conforme efectuará un agrupamiento cambiante a lo largo de la misma, propiciado así la flexibilidad necesaria para un acertado tratamiento de la variabilidad del grupo en cuanto a necesidades a atender.

- Con relación al espacio del alumno en el aula, cabe mencionar la total autonomía para ubicarse en el mismo, no teniendo un espacio-fijo o asignado a modo de pupitre o posición establecida. Así, en función de la actividad a realizar (individual, en pareja, en grupo) éste ocupa aquel lugar que desee siempre que se 
adecue a la proximidad (por motivo de la actividad conjunta o guiada) o distanciamiento (a efectos de no molestarse unos a otros) que se requiera $(\mathrm{CC}$, AL8). De igual manera, no existen prescripciones acerca del mantenimiento de la posición sentada, pudiendo deambular por el aula en función del interés que despierte una u otra actividad o de la necesidad de contacto con el maestro (CC, AL9).

- El espacio del docente en el aula tampoco está establecido. Si bien pasa la mayor parte de la sesión delante del espejo con las actividades de moldeamiento fonoarticulatorio, aprovecha los momentos iniciales y finales para aproximarse a otros espacios del aula que permitan el acercamiento al grupo y promuevan la comunicación espontánea y global de éste (mesa y sillas alrededor, centro del aula en coro, etc.), (CC, AL2).

\section{Recursos materiales}

Con objeto de apreciar la importancia otorgada por los profesionales AL del estudio hacia los recursos materiales como elementos condicionantes de su actuación docente, se observa que ningún profesional considera los recursos materiales como el principal factor sobre el que haya que incidir para mejorar la función que vienen desempeñando (C, AL3).

Analizando los materiales que estos profesionales utilizan en su aula, cabe citar la existencia de determinados programas editados como base para el desarrollo de los diversos componentes lingüísticos ( $\mathrm{AD}, \mathrm{AL2}$ ), aunque a veces se valen de materiales de fabricación casera para trabajar aspectos fonéticos (sopladores, lengüetas, globos) o semánticos (folleto de compras del supermercado), exigiendo de este personal la dedicación oportuna para su diseño y elaboración:

- "Me consta que trae el coche lleno de cosas que ella misma crea y adapta." (E, DD)

- "En realidad, son materiales ya elaborados, donde aparecen las fichas seleccionadas en función del fonema que vas a trabajar y la posición dentro de la palabra, o si es relativo a los otros compontes, el aspecto que vamos a cubrir con la sesión: actividades, juegos de expresión, dibujos que utilizamos en las representaciones de los cuentos para trabajar vocabulario y a partir de ahí hacer actividades de denominación, identificación [...] Tú les dices la palabra y ellos identifican, o que ellos denominen ¿eh?, etc.”(E, AL3) 
También son asiduos a la utilización de programas informáticos que van surgiendo y que, según opina este profesorado, comienzan a abarcar multitud de facetas relacionadas con su materia - “y que están teniendo éxito.”- (E, AL7); (CC, AL9).

- "Te estoy hablando de programas informatizados que ahora mismo, del mercado no tengo apenas si no son los que yo me estoy buscando de Internet a través de la red." (E, AL2).

En esta tipología de materiales hay profesionales que comentan la escasez de programas elaborados en el mercado de cara a promover componentes no fonéticos -como el pragmático o el morfosintáctico-, y la necesidad de una mayor implicación por parte de los profesionales que deseen dotar su trabajo de un carácter más global desde el punto de vista del desarrollo lingüístico del alumno (CC, AL6).

- "Demando más programas específicos que no hay en el mercado, porque todo se centra al aspecto de fonética y fonología. Y entonces, hay una carencia de un componente muy importante que es el componente pragmático y el componente morfosintáctico, que a fin de cuentas es el que más necesitan los niños." (E, AL6).

En este sentido, cabe advertir que, en el conjunto del sistema educativo de la Sociedad del Conocimiento, comienza a demandarse la generalización del uso de recursos asociados a las nuevas tecnologías (medios audiovisuales, ordenadores y software, etc.) para lo cual este profesional encuentra la dificultad asociada a la dependencia de la ubicación y disponibilidad en cada centro de los soportes/hardware. En cualquier caso, dichos profesionales participan directamente en la selección de los materiales que utilizan, siendo en múltiples ocasiones los diseñadores de los mismos al tener que ser adaptados o inventados en función de las necesidades específicas del alumnado (CC, AL3); (CC, AL4); (AD, AL7).

- "En muchos casos, tenemos que construir los materiales que vamos a utilizar con nuestros alumnos e intentar adaptar cosas cotidianas por motivos económicos y también por su facilidad de obtención "(E, AL6) 


\section{Discusión y Conclusiones}

A la vista de los resultados expuestos, podemos señalar que la labor del maestro de audición y lenguaje -en los casos que estudiados-, si bien se aproxima a los planteamientos que propugnan autores como Acosta (2005) o Moreno y Leal (2005), en cuanto a la promoción de una modalidad de apoyo curricular multidimensional, se haya distante de un enfoque integral colaborativo, como el defendido por Hodson y Paden (1991) o Fey y Cleave (1997). En este sentido, se haría preciso un enfoque más global e interactivo del lenguaje entre el conjunto de intervinientes en la labor educativa del alumno (resto de profesores, familiares) que se centre en facilitar la comunicación social en el conjunto de los contextos en los que se desarrolla su acción, en la línea de lo propuesto por Brinton, Fujiki y Powell (1997).

\section{Conclusiones}

Analizando los resultados en los diversos aspectos sobre los que se centra esta investigación podemos señalar las siguientes conclusiones:

\section{Modelo de intervención de corte reproductivo}

El eje principal de su actuación se centra en procedimientos reproductivos-repetitivos en los que predomina la actuación del docente. No se trabajan los contenidos del resto de las áreas ni se suelen encargar actuaciones para el aula ordinaria (escasa colaboración en casa y ocasional con el profesional de pedagogía terapéutica). Se aprecia que la secuencia de actividades-tipo es semejante en las distintas sesiones y centros -lo cual no impide que improvise en el desarrollo de las mismas e intente fomentar algo de creatividad al final de las sesiones-, al no existir una planificación detallada e inserta en el currículo. No ha sido posible encontrar evidencias de intervención en donde se observen los rasgos del modelo teórico sustentador de la creación del perfil, que lo era de prioridad asesora e intervención horizontal no terapéutica.

\section{Metodología: actividades y tareas}

Realiza actividades individuales o en pareja, fuera del aula ordinaria, sin apenas planteamientos colaborativos con el resto del profesorado (salvo algo con el maestro de pedagogía terapéutica) y padres (estos no se prestan en su mayoría), con un enfoque dirigido 
exclusivamente a tratar la problemática específica (logopédica) diagnosticada en el informe de valoración (alumnado con necesidades educativas especiales). La presión del entorno de enseñanza tradicional se superpone a los principios innovadores en la determinación de tareas y actividades que el maestro itinerante de audición y lenguaje desarrolla con sus alumnos. Tareas horizontales de carácter colaborativo no tiene apenas cabida en su panel de tareas.

\section{Estilo de enseñanza y aprendizaje}

Intenta considerar el estilo de aprendizaje de cada alumno y los canales de motivación a la hora de plantear las actividades a realizar (variedad de materiales, tipo de refuerzo/recompensas, etc.), apreciándose su esfuerzo en desarrollar una labor lo más didáctica y educadora que le permite el contexto de aprendizaje y la tarea a desarrollar, llevando a cabo una secuenciación individual de las actividades que mantenga el orden de adquisición natural en función de los objetivos planteados y adecuando las estrategias a las necesidades de cada alumno.

\section{Componente prioritario: fonoarticulatorio}

Nos encontramos con una metodología centrada en las intervenciones secuenciales fonoarticulatorias, si bien hay mayor amplitud de contenidos trabajados -en cuanto a tipología y componentes lingüísticos-, la prevalencia de estos es muy relevante.

\section{Insuficientes recursos humanos}

Se evidencia escasez de recursos humanos para desarrollar cualquiera de los modelos de intervención que se plantean, si bien dadas las exigencias colaborativas y de tareas no lectivas del modelo curricular, éste se ha visto perjudicado aún más en sus posibilidades de promoción. Se expresa una inexistencia de períodos de dedicación a los centros/comunidades educativas para tareas de planificación/coordinación/atención, etc., dificultando su integración en los mismos y la eficacia de su función, a lo cual se une el desinterés familiar y la concepción como especialista-externo por parte de los centros.

Aunque las demandas se centran en la cantidad de recursos, comienza a advertirse ciertos requerimientos en cuanto a la calidad y tipología de su actuación, lo cual confirma la valoración de actuaciones de carácter curricular-global y las exigencias y reconocimientos que conlleva su labor itinerante. 


\section{Recursos materiales adecuados}

Se evidencia una satisfacción por los materiales disponibles, si bien se reconoce la exigencia de disponibilidad de vehículo propio y el necesario traslado de los mismos dificulta la organización efectiva de dichos recursos en cada espacio de centro. En este sentido, podemos reseñar que a lo largo de este estudio, al igual que señalaba Fey (1986) en los resultados de sus investigaciones, se aprecia la preeminencia de procedimientos rehabilitadores clínicos provenientes de la medicina y de la psicología que se centran en la realización de evaluaciones e intervenciones sucesivas, las cuales si bien ayudan a superar determinados problemas -sobre todo de carácter articulatorio-, no conectan las habilidades lingüísticas enseñadas con situaciones reales de comunicación y de enseñanza-aprendizaje.

Se hace necesaria, la potenciación de planteamientos colaborativos donde los distintos profesionales del centro que atienden a este tipo de alumnado (maestro de audición y lenguaje y resto de profesores de aula ordinaria o del aula de apoyo) analicen el lenguaje en sus contextos de producción y busquen soluciones compartidas, pasando de un modelo de evaluación centrado en el déficit por otro más encauzado al proceso. Esta forma de llevar a cabo la actuación que comentamos posibilitará, en la línea de lo planteado por McCauley y Fey (2006), que a través de la mediación educativa se proporcionen los apoyos necesarios para que el niño utilice el lenguaje de manera global favoreciendo el uso de estrategias para conseguir otros aprendizajes dentro del aula.

Con relación al papel de la familia, consideramos que es otro de los aspectos a potenciar para asegurar la eficacia de este modelo de trabajo, debiendo facilitar, mediante la formación y coordinación con la misma desde los centros escolares, que ésta pueda incorporarse plenamente al proceso de evaluación e intervención en el lenguaje, de manera que se armonicen, como señala Turnbull, Turnbull y Kyzar (2009), las actividades llevadas a cabo en las aulas con las que los niños desarrollan en las casas.

\section{Prospectivas de futuro}

Sería conveniente continuar en esta línea y corroborar lo aquí analizado con múltiples estudios de casos en diversos contextos educativos que enriquezcan y delimiten lo aquí obtenido, presentando a continuación posibles ideas o vías de investigación sobre las cuales sería interesante trabajar en el futuro, con objeto de continuar analizando el pensamiento 
profesional y la actuación educativa desarrollada por parte de estos profesionales, de cara a profundizar en el conocimiento de las posibilidades educativas relacionadas con dicha temática.

a) ¿Qué estrategias de enseñanza en equipo pueden desarrollarse en los centros de forma que el ámbito lingüístico se impulse asegurando un enfoque multidisciplinar?

b) ¿Qué programas de actuación pueden diseñarse de cara a incardinar en el currículo la atención a la diversidad en lo comunicativo-lingüístico y qué papel puede desempeñar el AL de centro en su desarrollo?

\section{Referencias}

Acosta-Rodríguez, V.M. (2003). Las prácticas educativas ante las dificultades del lenguaje. Una propuesta desde la acción. La colaboración entre logopedas, psicopedagogos, profesores y padres. Barcelona: Grupo Ars XXI de Comunicación, S.A.

Acosta-Rodríguez, V.M. (2004). Las prácticas educativas ante las dificultades del lenguaje. Una propuesta desde la acción. Barcelona: STM Editores.

Acosta-Rodríguez, V.M. (2005). Evaluación, intervención e investigación en las dificultades del lenguaje en contextos inclusivos. Revisión, resultados y propuestas. Revista de Logopedia, Foniatría y Audiología, 25(4),148-161.

Acosta-Rodríguez, V.M. (2006a). Efectos de la intervención y el apoyo mediante prácticas colaborativas sobre el lenguaje del alumnado con necesidades educativas específicas. Revista de Logopedia, Foniatría y Audiología, 26(1), 36-53.

Acosta-Rodríguez, V.M.(2006b). El papel del lenguaje en la escolarización de todo el alumnado: revisión, desafíos y propuestas. En E. Mendoza Lara (Ed.), Actas XXV Congreso Internacional de AELFA (pp.53-65). Granada: Universidad de Granada.

Acosta-Rodríguez, V.M. y Moreno-Santana, A. M. (1999). Dificultades del lenguaje en ambientes educativos. Del retraso al trastorno específico del lenguaje. Barcelona: Masson.

Acosta-Rodríguez, V.M. y Moreno Santana, A. M. (2003). Dificultades del lenguaje: una perspectiva educativa. Bordón, 55(1), 51-60.

Bloom, L. y Lahey, M. (1978). Language development and language disorders. New York: Wiley y Sons.

Brinton, B., Fujiki, M., y Powell, J. (1997). The ability of children with language impairment to manipulate topic in a structured task. Language, Speech and Hearing Services in Schools, 28, 3-11. 
Castejón-Fernández, L.A. y España-Ganzaráin, Y. (2004). La colaboración logopeda-maestro: hacia un modelo inclusivo de intervención en las dificultades del lenguaje. Revista de Logopedia, Foniatría y Audiología, 24, 55-66.

Cooper, J., Moodley, M. y Reynell, J. (1982). Método para favorecer el desarrollo del lenguaje. Un programa de desarrollo para los niños con una problemática del lenguaje en edades tempranas. Barcelona: Médica y Técnica.

Crystal, D. (1981). Lenguaje infantil, aprendizaje y lingüística. Barcelona: Médica y Técnica.

Dubois, M. E. (1991). El proceso de lectura: de la teoría a la práctica. Buenos Aires: Aique.

Fey, M. (1986). Language intervention with young children. Needham Heights, MA: Allyn \& Bacon.

Fey, M., Catts, H. y Larrive, L. (1995). Preparing preschoolers for the academic and social challenges of school. En S. Warren y J. Reichle (Eds.), Communication and language intervention series, vol. 5, Language intervention: Preschool through the elementary years (pp. 3-37). Baltimore: Paul H. Brookes P.

Fey, M., y Cleave, P. (1997). Two models of grammar facilitation in children with language impairments. Journal of Speech. Language, and Hearing Research, 40, 5-19.

Gallardo-Ruiz, J.R. y Gallego-Ortega, J.L. (1993). La intervención del lenguaje. En J.R. Gallardo Ruiz y J.L. Gallego Ortega (Eds.), Manual de Logopedia Escolar. Un enfoque práctico. Málaga: Ediciones Aljibe.

Goetz, J.P. y Le Compte, M.D. (1988). Etnografía y diseño cualitativo en investigación educativa. Madrid: Morata.

Hemmeter, M. y Grisham-Brown, J. (1997). Developing children's language skills in inclusive early childhood classrooms. Dimensions of Early Childhood, 25, 6-13.

Hodson, B., y Paden, E. (1991). Targeting intelligible speech: A phonological approach to remediation. Austin, TX: PRO-ED.

Law, J., Boyle, J., Harris, F., Harkness, A., y Nye, C. (2000). The relationship between the natural history and prevalence of primary speech and language delays: Findings from a systematic review ofthe literature. International Journal of Language and Communication Disorders, 35, 165-188.

Lindsay, G. y Dockrell, J. (2002). Meeting the needs of children with speech language and communication needs: a critical perspective on inclusion and collaboration.ChildLanguageTeaching and Therapy, 18, 91-101.

Manolson, A. (1992). It Takes Two to Talk. Toronto: The Hanen Centre.

McCauley, R., \& Fey, M. (2006). Treatment of language disorders in children. Baltimore: 
Paul. H. Brookes P.

Monfort, M. (1983). La intervención logopédica. Cuadernos de pedagogía, 101, 20-26.

Monfort, M. (1990). Logopedia. Cuadernos de pedagogía, 178, 84-86.

Leal Hernández, E. y Moreno Santana, A.M. (2005). Estudio de las prácticas educativas con niños con trastorno específico del lenguaje. Revista de logopedia, foniatría y audiología (4)25, 174-189

Moya-Maya, A. (2002). El profesorado de apoyo ¿dónde? ¿cómo? ¿cuándo?... realiza su trabajo. Málaga: Ediciones Aljibe.

Muntaner-Guasp, J.J. (2000). Aportaciones de la Educación Especial a las escuelas eficaces. En A. Miñambres-Abad y G. Jové-Monclús (Eds.), La atención a las necesidades educativas especiales: De la educación infantil a la universidad (pp.77-93). Lleida: Ediciones de la Universidad de Lleida.

Parrilla-Latas, A. (1996). Apoyo a la escuela: un proceso de colaboración. Bilbao: Mensajero.

Prelock, P.(2000). An Intervention Focus for Inclusionary Practice. Language, Speech\&Hearing Services in Schools, 31, 296-298.

Richards, J.C. y Rodgers, T.S. (1986). Approaches and methods in language teaching: A description and analysis. Cambridge: Cambridge University Press.

Rodríguez-Gómez, G., Gil-Flores, J. y García-Jiménez, E. (1996). Metodología de la Investigación Cualitativa. Málaga: Ediciones Aljibe.

Stake, R.E. (2007). Investigación con estudio de casos .Madrid: Morata.

Taylor, S.J. y Bogdan, R. (1986). Introducción a los métodos cualitativos de investigación. Barcelona: Paidós.

Turnbull, A., Turnbull, H. y Kyzar, K. (2009). Cooperación entre familias y profesionales como fuerza catalizadora para una óptima inclusión: enfoque de los Estados Unidos de América. Revista de Educación, 349, 69-99. DOI: 10-4438/1988-592X-RE-2012-363175.

Vigotsky, L. (1995). Pensamiento y lenguaje. Buenos Aires: Paidós

Wiig, E. H., (2001). Valoración del lenguaje basada en la actuación del alumno en los colegios. Introducción de nuevas herramientas para la observación del comportamiento y planificación de la intervención. Revista de Logopedia, Foniatría y Audiología, 21,24- 30.

Woods, P. (1989). La escuela por dentro: la etnografía en la investigación educativa. Barcelona: Paidós. 